\title{
Guidewire-Induced Carotid Cavernous Fistula
}

\author{
T.-S. KIM, M. EZURA, A. TAKAHASHI*, S. NISHIMURA, T. YOSHIMOTO* \\ Department of Neurosurgery, Kohnan Hospital; Sendai, Japan \\ * Department of Neurosurgery, Tohoku University; Sendai, Japan
}

Key words: guidewire, carotid cavernous fistula, embolization, aneurysm

\section{Summary}

A rare case of carotid cavernous fistula occurring during endovascular embolization of the left carotid cave aneurysm in a 48-year-old female is reported. It was thought to be caused by the tear of a small branch derived from the intracavernous internal carotid artery while the guidewire was passing the sharp posterior bend of the intracavernous internal carotid artery. The left carotid cave aneurysm was completely occluded with five Guglielmi detachable coils assisted by neck plasty technique. It was decided to follow-up the carotid cavernous fistula since it was asymptomatic. Follow-up angiogram performed two weeks later revealed spontaneous obliteration of the carotid cavernous fistula.

\section{Introduction}

Traumatic carotid-cavernous fistula has been reported to occur secondary to trauma of several types ${ }^{7}$. Recent advances in neuroendovascular techniques and microcatheters made it possible to access the complex cerebrovascular lesions. However it may also increase the risk of unexpected vascular injuries. We present a case of guidewire-induced carotid cavernous fistula as an unusual complication of endovascular therapy and the possible mechanism is discussed.

\section{Case Report}

A 48-year-old right handed female was referred to our hospital with Hunt-Hess grade II subarachnoid hemorrhage. Computed tomographic scan on admission showed subarachnoid hemorrhage localized mainly in the perimesencephalic cistern and right basal cistern. Anterior communicating artery aneurysm was suspected on cerebral angiogram. In addition, an incidental $5 \mathrm{~mm}$ left carotid cave aneurysm was present (figure 1). We thought that the origin of subarachnoid hemorrhage might be the anterior communicating artery aneurysm and performed exploratory craniotomy. At surgery no aneurysm could be found in the anterior communicating territory or in the right middle cerebral, anterior cerebral, internal carotid, basilar and superior cerebellar arteries. We concluded that she had a perimesencephalic subarachnoid hemorrhage of unknown origin and performed Guglielmi detachable coil embolization of the carotid cave aneurysm one month later.

An 8-French guiding catheter (Toray, Tokyo, Japan) was advanced into the left cervical internal carotid artery. A balloon catheter, a silastic balloon attached to the Tracker-18 Unibody catheter (Boston Scientific, Watertown, MA), was introduced for neck plasty technique. This maneuver was assisted by a Tracker-16 Flextip guidewire (Boston Scientific) and it was placed 
across the aneurysm neck. Then a microcatheter (Prowler 10, Cordis, Miami, FL) was advanced coaxillary into the petrous portion of internal carotid artery over a guidewire (GT12, Terumo, Tokyo). While the guidewire was passing a sharp posterior bend of the intracavernous internal carotid artery, fluorogram showed the tip of the guidewire was located just outside the vessel wall (figure 2). Left internal carotid angiogram performed immediately after this procedure showed carotid cavernous fistula. The vital signs were stable and no neurological deficits or signs of carotid cavernous fistula occurred. We continued the procedure. A Prowler 10 microcatheter was advanced into the aneurysm. After a good positioning was confirmed, the balloon was inflated across the neck of aneurysm. Five coils (GDC soft $10,4 \times 6,3 \times 8,2 \times 6,2 \times 4,2 \times 2)$ were detached by temporary balloon protection without complication. The angiography demonstrated complete occlusion of the aneurysm with excellent parent artery flow. We decided to follow-up the carotid cavernous fistula since it was asymptomatic.

Follow-up angiogram performed one week later showed still patent fistula (figure 3 ). We decided to occlude it by transvenous embolization two weeks after first embolization of the aneurysm. However control angiogram before transvenous embolization demonstrated spontaneous obliteration of the carotid cavernous fistula (figure 4).

\section{Discussion}

Since 1991, endovascular treatment of cerebral aneurysm using Guglielmi detachable coil (GDC) system has become an alternative method to surgical clipping in selected patients. The procedure-related complications have been reported to be $5 \sim 13 \%$ during endovascular surgery for cerebral aneurysm ${ }^{2,4,9}$. The procedure-related complications include vascular perforation or injury by catheter or guidewire, obstruction of parent or distal artery by coil herniation, and thromboembolism.

Halbach et $\mathrm{Al}^{6}$ reported 15 cases of vascular perforation caused by a catheter or guidewire from a series of 1200 endovascular procedures to treat vascular disorders involving the brain and spinal cord. The possible mechanisms of vascular perforations that they encountered during neurointerventional procedure included: mechanical perforation of a normal vessel, mechanical disruption of a dysplastic vessel or aneurysm, and fluid overinjection. Two deaths occurred among their series resulting from massive subarachnoid hemorrhage before definitive treatment. The perforation points in the other 13 cases were recognized and treated effectively with no episodes of rehemorrhage. When vascular perforation occurs during endovascular surgery it is important to recognize it immediately. Guidewire or catheter had better not be withdrawn from the perforation point. If the guidewire is the cause of perforation, advancing the catheter over it may seal the hole. This can then be occluded by adequate embolic materials. This method might be useful for perforation by guidewire. However, this might also carry some risk because it will result in enlargement of the hole. In our case, the guidewire was withdrawn from the perforation point. Carotid cavernous fistula was recognized but not progressed. The vital signs were stable and any symptoms and signs of fistula were not developed until spontaneous obliteration. Perforation of a normal vasculature by a guidewire or catheter may have a better result than mechanical disruption of a dysplastic vessel or aneurysm because the muscular wall of a normal artery can contract to seal a small perforation.

Barr et $\mathrm{Al}^{1}$ reported a similar case to ours of carotid-cavernous fistula occurring after embolization of a cavernous sinus meningioma. The patient had experienced considerable headache and nausea during catheterization and the procedure was discontinued. A followup internal carotid artery arteriogram did not show any appreciable change. On the next day, arteriogram showed carotid-cavernous fistula. They thought the cause of carotid-cavernous fistula might have been perforation by the guidewire during catheterization of meningohypophyseal trunk at the sharp bend of its origin. However, they did not confirm perforation by guidewire on angiogram. In our case, the site of perforation was at the sharp posterior bend of the intracavernous internal carotid artery. Perforation occurred while the guidewire was passing the sharp bend and confirmed by continuous recording by fluoroscopy.

Spontaneous obliteration of traumatic carotid cavernous fistula has been considered 


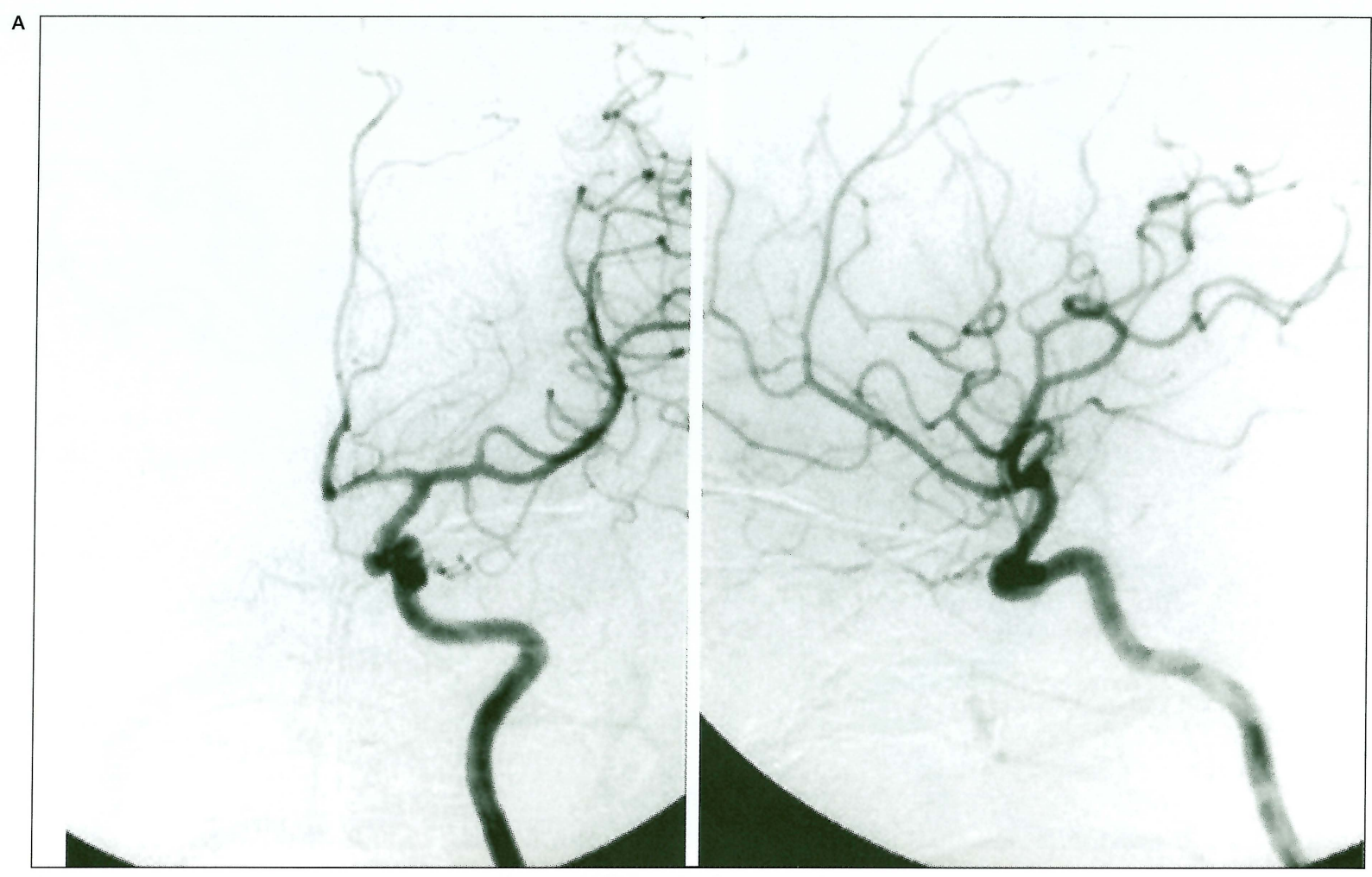

Figure 1 Anteroposterior (A) and lateral (B) left internal carotid angiograms show a $5 \mathrm{~mm}$ carotid cave aneurysm.
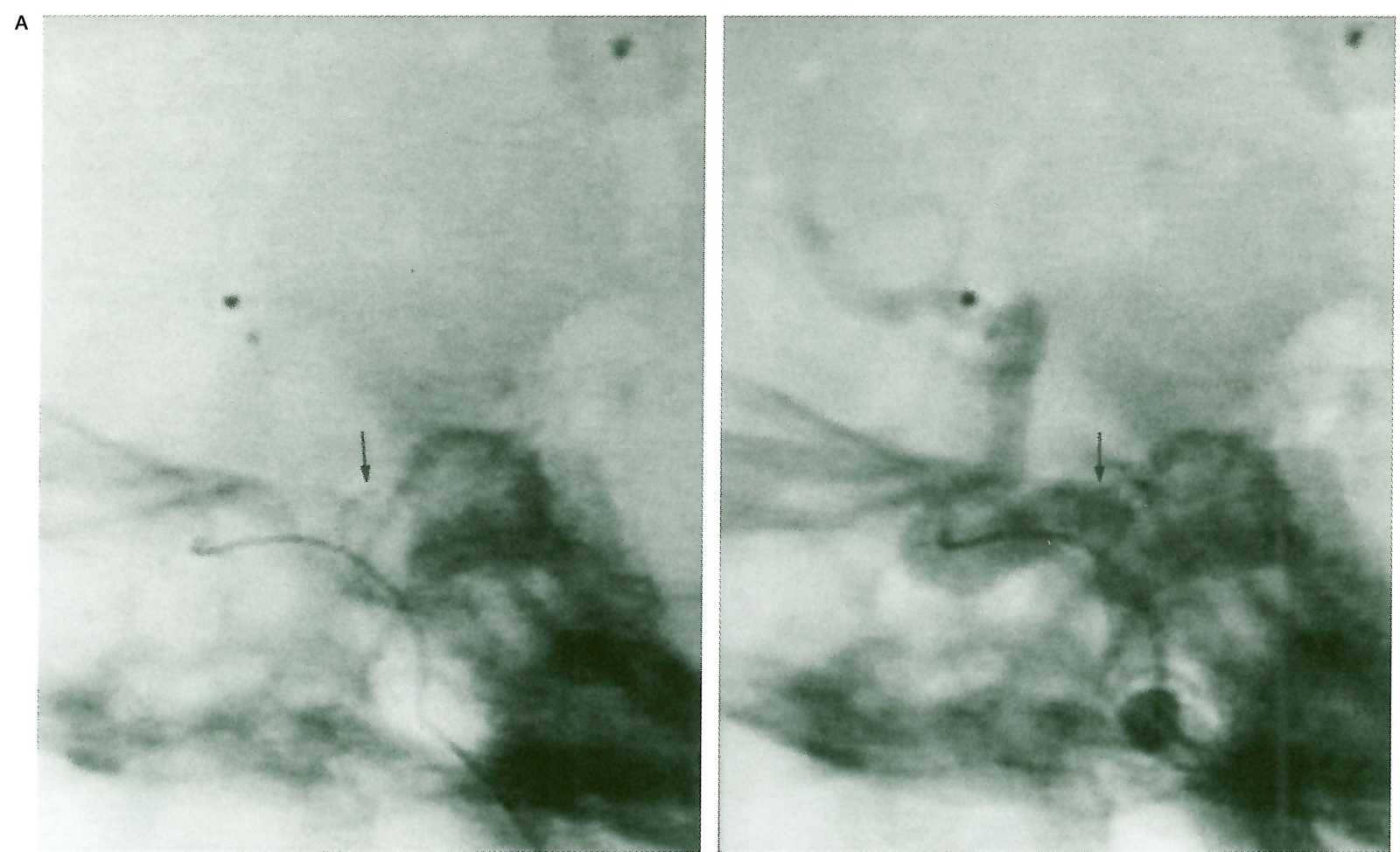

Figure 2 A guidewire is noted to leave the vascular system in the left intracavernous internal carotid artery during navigation of left internal carotid artery (arrow). 


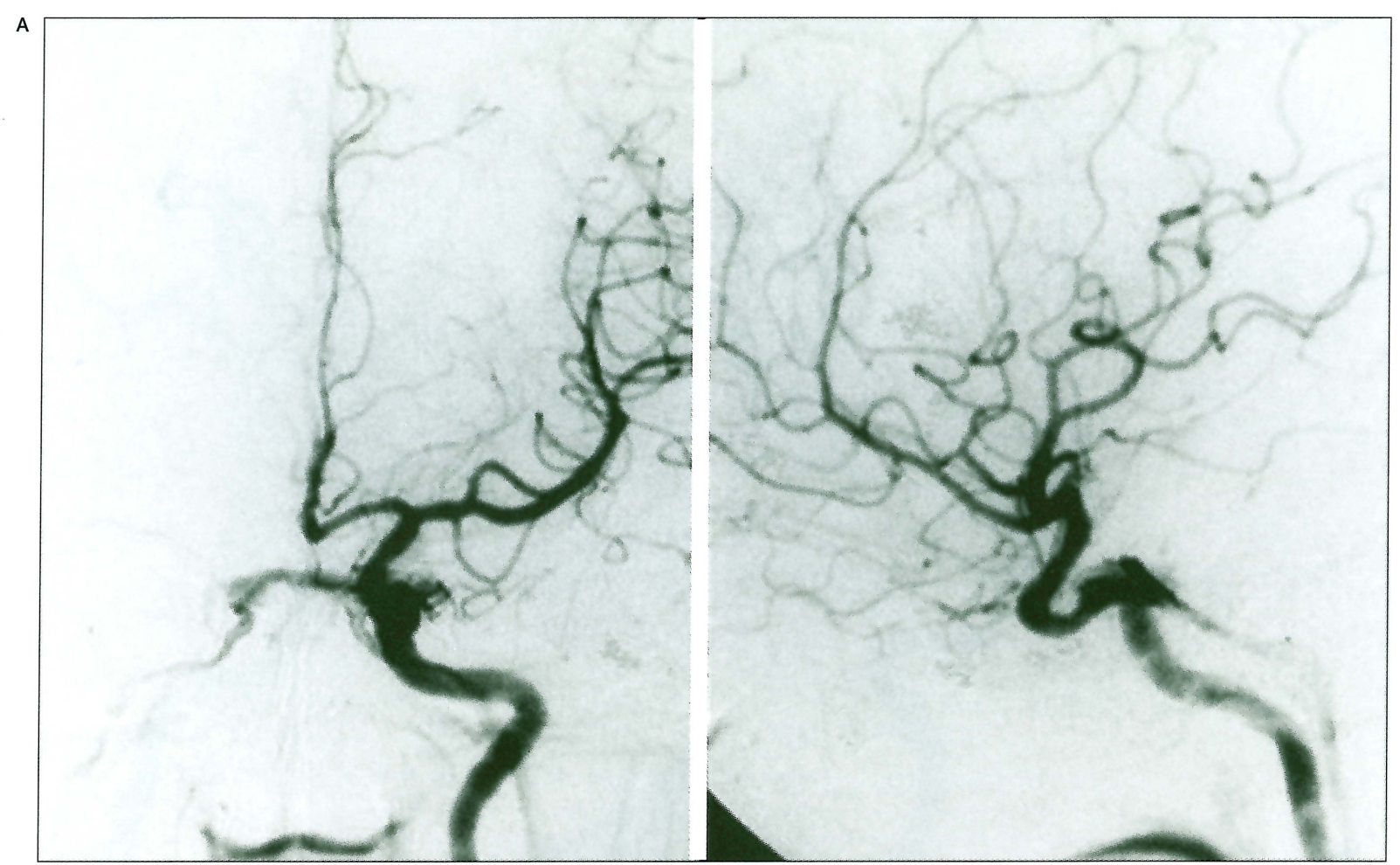

Figure 3 Follow-up cerebral angiogram obtained one week after GDC embolization of carotid cave aneurysm shows still patent carotid-cavernous fistula and complete occlusion of the carotid cave aneurysm.

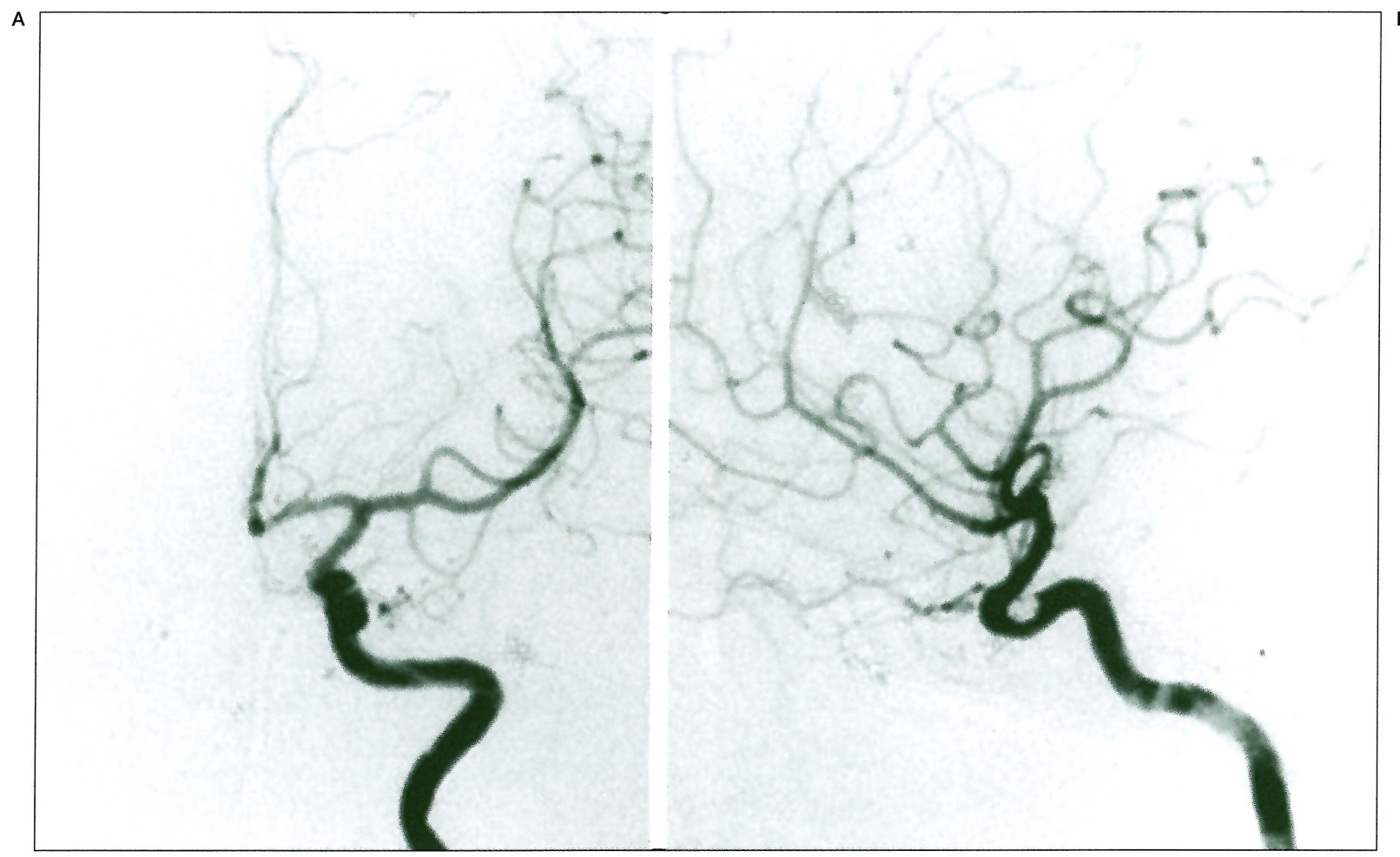

Figure 4 Control cerebral angiogram before transvenous embolization demonstrates the complete obliteration of carotidcavernous fistula. 
uncommon and its possible mechanisms include embolism of fibrin platelet clots, fall of blood pressure, and reaction to contrast media ${ }^{3,5,8}$. In our cases, follow-up angiogram performed one week later showed still patent fistula. We decided to treat by transvenous embolization. However, control angiography before transvenous embolization demonstrated spontaneous obliteration of carotid cavernous fistula.

It is important for the endovascular surgeon to recognize the possibility of iatrogenic vascular injury during endovascular surgery. Early detection and management of this complication is essential for a good outcome.

\section{References}

1 Barr JD, Mathis JM, Horton JA: Iatrogenic carotid-cavernous fistula occurring after embolization of a cavernous sinus meningioma. Am J Neuroradiol 16: 483485, 1995.

2 Bavinzski, Killer $\mathrm{M}$ et $\mathrm{Al}$ : Treatment of basilar artery bifurcation aneurysm by using Guglielmi detachable coils: a 6-year experience. J Neurosurg 90: 843-852, 1999.

3 Canova A, Esposito S et Al: Spontaneous obliteration of a carotid-cavernous fistula associated with fibromuscular dysplasia of the internal carotid artery. J Neurosurg Sci 31(1): 37-40, 1987.

4 Debrun G, Aletich VA et Al: Selection of cerebral aneurysms for treatment using Guglielmi detachable coils; the preliminary university of Illinois at Chicago experience. Neurosurgery 43(6): 1281-1297, 1998.

5 Ferrera PC: Traumatic carotid-cavernous sinus fistula with spontaneous resolution. Am J Emerg Med 15(4): 386-388, 1997.

6 Halbach VV, Higahida RT et Al: Management of vascular perforations that occur during neurointerventional procedure. Am J Neuroradiol 12: 319-327, 1991.

7 Lewis AI, Tomsick TA, Tew JM: Management of 100 consecutive direct carotid-cavernous fistulas: results of treatment with detachable balloons. Neurosurgery 36(2): 239-245, 1995.
8 Nishijima M, Iwai R et Al: Spontaneous occlusion of traumatic carotid cavernous fistula after orbital venography. Surg Neurol 23(5): 489-492, 1985.

9 Solander S, Ulhoa A et Al: Endovascular treatment of multiple intracranial aneurysms by using Guglielmi detachable coils. J Neurosurg 90: 857-864, 1999. 